\title{
LA FORMACIÓN EN INTERPRETACIÓN REMOTA: UNA EXPERIENCIA DOCENTE INTERUNIVERSITARIA
}

\author{
REMOTE INTERPRETING TRAINING: \\ AN INTERCOLLEGIATE EXPERIENCE
}

https://dx.doi.org/10.15304/ie.29.6271

Francisco J. Vigier-Moreno

Universidad Pablo de Olavide de Sevilla (UPO)

fvigier@upo.es

Raquel Lázaro Gutiérrez

Universidad de Alcalá (UAH)

raquel.lazaro@uah.es

\section{RESUMEN}

La interpretación remota, es decir, la mediación lingüística oral que se realiza a través de algún medio tecnológico (como la interpretación telefónica o la interpretación por videoconferencia), está cobrando gran impulso en todo el mundo debido a sus incuestionables ventajas en cuanto a coste y disponibilidad tanto geográfica como temporal de los intérpretes. Sin embargo, los centros universitarios de formación de intérpretes no parecen estar ofreciendo programas formativos en esta nueva modalidad de trabajo. Para cubrir este vacío, en los cursos académicos 2016-17, 2017-18 y 2018-19 se llevó a cabo una experiencia docente interuniversitaria en forma de taller de interpretación remota entre la Universidad Pablo de Olavide de Sevilla y la Universidad de Alcalá, cuyo diseño, implementación, metodología y evaluación se describen en este artículo.

Palabras clave: interpretación remota; formación de intérpretes; metodología docente.

\section{ABSTRACT}

Remote interpreting, that is, oral language mediation undertaken through technological means (such as telephone interpreting and videoconference interpreting), is gaining momentum all over the world thanks to its unquestionable advantages in terms of cost and availability of interpreters, both geographic and temporal. Nevertheless, interpreter training schools seem to not offer training programmes in this new working mode in interpreting. With the aim of filling this gap, an intercollegiate workshop in remote interpreting was held in the academic years 2016-17, 2017-18 and 2018-19 for interpreter trainees from both the Universidad Pablo de Olavide of Seville and the University of Alcala. This paper describes

Recibido: 14/IX/2019. Aceptado: X/2019 
and discusses the design, implementation, methodology and assessment of this teaching experience.

Keywords: remote interpreting; interpreter training; teaching methodology.

\section{INTRODUCCIÓN}

La Interpretación Remota (en adelante, IR) es un término amplio que designa una forma de interpretación (es decir, de mediación interlingüística oral) realizada por medio de tecnologías de la información y la comunicación (Fantinuoli y Prandi, 2018: 164). Esta práctica interpretativa que se lleva a cabo fundamentalmente por teléfono o mediante videoconferencia está consolidándose en el mercado actual debido al desarrollo tecnológico y a las ventajas económicas y geográficas que reporta, por lo que se está convirtiendo, a su vez, en un nicho laboral con enorme proyección para los egresados de interpretación (García Luque, 2009: 27).

Aunque comparte con otras modalidades de interpretación muchas destrezas y habilidades (por ejemplo, competencia lingüística y habilidades de oratoria), los intérpretes remotos deben poseer una serie de competencias específicas para poder realizar su labor interpretativa satisfactoriamente sin estar en presencia física de los interlocutores, como aquellas relacionadas con la gestión de la comunicación remota. No obstante, las titulaciones actuales relacionadas con la formación universitaria de intérpretes no incluyen asignaturas concretas en IR ni tampoco parecen tener en cuenta esta modalidad en sus objetivos formativos (Ruiz Mezcua, 2018: 14).

Habiendo detectado esta realidad en los programas de grado dedicados a la formación de traductores e intérpretes de la Universidad Pablo de Olavide de Sevilla (en adelante, UPO) y la Universidad de Alcalá (en lo sucesivo, UAH), surge la necesidad de cubrir este vacío formativo ofreciendo a los estudiantes de estos centros experiencias formativas específicamente diseñadas para el desarrollo de competencias en esta modalidad interpretativa. En este artículo, tras describir brevemente la IR como actividad profesional y los principales principios de su enseñanza, presentamos una experiencia docente a partir de un taller interuniversitario desarrollado durante los cursos 2016-17, 2017-18 y 2018-19, centrándonos en su diseño, su implementación y los principales resultados obtenidos.

\subsection{Definición de la IR y relación con la Interpretación Bilateral}

En IR, los intérpretes precisan de la tecnología para poder conectarse con los hablantes primarios (Ruiz Mezcua, 2018: 10). Este tipo de interpretación se puede ofrecer por vía telefónica o por videoconferencia y se utiliza para tres modalidades: 1) bilateral (en la que el intérprete interviene tras los parlamentos de los usuarios, generalmente breves), que es la más frecuente; 2) simultánea (en la que el intérprete va transfiriendo a la otra lengua el discurso pronunciado por el orador original a la vez que este lo emite); y 3) consecutiva (en la que el intérprete reproduce, a partir de sus notas, el discurso que ha pronunciado el orador original una vez que este ha concluido su intervención).

La IR presenta distintas configuraciones. Se puede producir cuando los participantes primarios de una conversación están físicamente juntos y se conectan por teléfono o videoconferencia con 
un intérprete que está en otro lugar (como ocurre, por ejemplo, en la consulta de un médico en la que se presenta un paciente que no habla la lengua empleada por el profesional sanitario y se recurre a una interpretación telefónica por medio de un intérprete que no se encuentra físicamente en dicha consulta). En otras ocasiones, el intérprete sí se encuentra físicamente con uno de los usuarios y es el otro usuario el que se encuentra en una ubicación remota (por ejemplo, en una toma de declaración judicial internacional, en la que operador judicial e intérprete se encuentran juntos en la misma ubicación y el declarante es quien está en un lugar diferente). Y también puede darse que los participantes primarios no estén en el mismo sitio y se comuniquen mediante un intérprete que no se encuentre con ningunos de ellos sino en un tercer lugar, comunicándose mediante lo que se conoce como una llamada a tres, ya sea por teléfono o videoconferencia.

La modalidad de trabajo más frecuente en la IR es la interpretación bilateral (en adelante, IB), bien por vía telefónica bien por videoconferencia (Fernández Pérez, 2015: 261). La IB, que puede definirse como "aquella en la que el mismo intérprete interpreta a cada uno de los dos locutores cada pequeño fragmento de información desde y hacia las dos lenguas, tras la intervención de los mismos" (Foulquié Rubio, 2019: 20), reúne una serie de características que la distinguen de otras modalidades (Collados Aís y Fernández Sánchez, 2001). Así, a diferencia de la simultánea (que suele realizarse desde una cabina de interpretación), en la IB, de manera tanto presencial como remota, el intérprete establece contacto directo con los dos interlocutores. También a diferencia de la interpretación de conferencias, en la que los intérpretes suelen interpretar solo desde su lengua B o primera lengua extranjera hacia su lengua A o materna, en la IB el intérprete realiza la transferencia lingüística en ambas direcciones ${ }^{1}$. Si en la interpretación simultánea el intérprete va transmitiendo el mensaje en la lengua meta mientras aún se está enunciando el mensaje en la lengua origen, en la consecutiva clásica o de conferencias el intérprete reproduce en la lengua meta la totalidad del discurso enunciado en la lengua origen a partir de las notas que ha tomado. En la IB, en cambio, el intérprete va transmitiendo hacia la otra lengua los parlamentos (por lo general, no excesivamente extensos) de los interlocutores una vez que estos han concluido, lo que se conoce como consecutiva corta sin toma de $\operatorname{notas}^{2}$ (Pöchhacker, 2007: 19).

Otras características de la IB son la espontaneidad del discurso y la heterogeneidad situacional. Al contrario de lo que suele ocurrir en la interpretación de conferencias (sea simultánea o consecutiva), en la que los discursos suelen haberse preparado con anterioridad, en la IB los interlocutores suelen producir mensajes de manera espontánea. Así, aunque se trata, por lo general, de parlamentos más cortos - con ideas menos complejas - , suelen expresarse en un lenguaje igualmente menos elaborado $\mathrm{y}$, por ende, con frecuentes repeticiones, pausas, anacolutos, autocorrecciones, etc. (Collados Aís, Fernández Sánchez y De Manuel Jerez, 2001: 68-69). En cuanto a la situación comunicativa, también hay diferencias notables entre la IB en comparación con la interpretación de conferencias. Mientras que esta última modalidad suele producirse en un contexto de formalidad, como son los congresos

1 Lo más frecuente es que solo haya un intérprete, a excepción de los encuentros diplomáticos de primer nivel, en los que cada mandatario suele contar con su propio intérprete.

2 No obstante, el intérprete de IB tiene que dominar la técnica de la toma de notas porque, en caso de extenderse la intervención de uno de los interlocutores, serán las notas que haya tomado las que le faciliten la reproducción del discurso en la lengua meta. 
internacionales, las reuniones multilaterales internacionales o las institucionales supranacionales (por tanto, con una variedad habitualmente estándar de la lengua y un registro elevado), la IB tanto presencial como remota - se da en situaciones muy diversas en cuanto a formalidad, registro y variedades lingüísticas (por poner algunos ejemplos, en reuniones empresariales de alto nivel, en declaraciones policiales de detenidos o en una urgencia médica).

\subsection{Características específicas de la IR}

Los intérpretes remotos deben poseer una serie de habilidades específicas para ser capaces de interpretar de manera satisfactoria sin estar físicamente presentes en la interacción, ya que, sobre todo en la modalidad telefónica, no tienen acceso a gran parte de información contextual (qué individuos y otros elementos están presentes, cuáles son sus papeles, su lenguaje corporal, sus movimientos, etc.). A esto se suman las dificultades que surgen del uso de la tecnología, como una posible falta de cobertura, el uso de un equipo anticuado o defectuoso, un uso inadecuado del equipo, etc. (Pertusa Elorriaga, 2012: 27). Por lo tanto, la IR presenta ciertas características relacionadas con la falta o escasez de información visual, la coordinación del discurso, el uso de la tecnología, los protocolos que se deben seguir con cada cliente, las condiciones laborales, el estrés y el impacto emocional, y la ética.

Puede que uno de los aspectos más críticos y determinantes de la IR —en concreto, de la telefónica - sea la falta de información visual. Esta se ha pretendido mitigar recurriendo a la interpretación por videoconferencia, hasta el punto de que algunos autores afirman que es probable que esta última modalidad termine sustituyendo a la telefónica (González Rodríguez y Spinolo, 2017: 242).

Sin embargo, otros autores mencionan que la falta o escasez de información visual no afecta necesariamente de manera negativa ni a la comunicación (Braun, 2006) ni a la actuación del intérprete, ya que los intérpretes profesionales poseen habilidades para superar este tipo de dificultades (Ko, 2006) y se apoyan en la percepción del paralenguaje y otros aspectos como el tono de voz, la respiración de los hablantes, el ritmo o el volumen del discurso (Kelly, 2008; Creeze, 2013, Cheng, 2015). Del mismo modo, autores como Mikkelson (2003) o Lee (2007) señalan que la comunicación no verbal puede distraer al intérprete y, por otro lado, hay intérpretes que prefieren la modalidad remota porque les permite una mayor neutralidad.

La falta de información contextual también se debe a la ausencia, en la mayoría de los casos, de un contacto previo con el intérprete en el que se le explique la situación comunicativa. La IR se usa especialmente en situaciones en las que se requiere la intervención de un intérprete de manera urgente e inmediata, como en el servicio de urgencias de un hospital o para una asistencia en carretera en caso de un siniestro (Lázaro Gutiérrez, 2019). Por tanto, es frecuente que el intérprete reciba el encargo de IR sin conocer la situación comunicativa en la que se necesita su mediación, teniendo que enfrentarse a una primera dificultad de "contextualizar el encuentro" (Fernández Pérez, 2015: 266). De ahí que el intérprete que se dedique a la IR deba desarrollar una gran capacidad de adaptación.

Además de la escasez de información visual, la coordinación del discurso entre los participantes, típica de la IB, presenta peculiaridades en el caso de la IR. De hecho, es posible que 
los intérpretes remotos confundan los silencios de los hablantes con fallos tecnológicos, ya que no son capaces de ver con claridad sus caras y percibir que están haciendo una pausa. Asimismo, el uso de la tercera y primera persona es controvertido, ya que, aunque se suela defender que los intérpretes deben usar la primera persona (Bot, 2005; Kelly, 2008), en la modalidad remota se hace necesario utilizar la tercera persona, sobre todo cuando surgen dificultades y malentendidos y los intérpretes tienen que intervenir para pedir o realizar aclaraciones (Rosenberg, 2004; Hsieh, 2006; Lee, 2007).

Wadensjö (1999), por su parte, realizó un estudio exhaustivo de la coordinación en conversaciones mediadas por intérpretes y averiguó que las imprecisiones en la interpretación se producen por tres razones principales: 1) los intérpretes no son capaces de controlar la distribución de turnos de habla; 2) los intérpretes no son capaces de retener fragmentos largos de discurso; y 3) los intérpretes necesitan interrumpir para pedir aclaraciones. Además, probó que a los intérpretes les cuesta más controlar los turnos de habla si no están presentes físicamente y es necesario que adopten un papel más activo, algo que también corroboró Hsieh (2006).

Fernández Pérez $(2015,2017)$ también identificó la capacidad de coordinación entre aquellas habilidades que los intérpretes remotos deben adquirir. Esta coordinación implica gestionar el principio y final del encuentro, organizar los turnos de habla e interrumpir a los hablantes primarios cuando sea necesario. Dentro de la tarea de coordinación del intérprete se encuentran las siguientes acciones: 1) presentarse adecuadamente a todos los participantes en la conversación para tener la oportunidad de explicar su papel y su código ético; 2) sugerir unas pautas básicas para el desarrollo de la conversación (como evitar los solapamientos, hablar directamente al interlocutor usando la primera persona, no dirigirse directamente al intérprete a no ser que sea necesario transmitir información contextual o se haya detectado que existe algún malentendido, etc.) (Lázaro Gutiérrez y Cabrera Méndez, 2019); y 3) explicar que todo lo que se diga será confidencial y se traducirá, y que el intérprete podrá intervenir en caso de que haya algún malentendido o sea necesario clarificar algún detalle.

El uso de la tecnología es otro elemento clave de la IR. La proliferación de los teléfonos móviles y la videoconferencia, el descenso de los costes de telefonía, la mejora en la calidad de las conexiones y la inmediatez con que se puede proporcionar interpretación en una gran variedad de lenguas son tres de los factores que han hecho que la interpretación telefónica sea tan popular hoy en día. Sin embargo, aunque la tecnología mejora día a día haciendo posible una comunicación más frecuente y eficiente, la calidad del sonido ha sido reiteradamente una preocupación y un objeto de estudio (Lee, 2007; Kelly, 2008). Con el uso de los teléfonos móviles, los usuarios de IR suelen utilizar la función de manos libres antes que otros sistemas, como el uso de cascos duales o pasarse el auricular del teléfono de un hablante a otro, lo que tiene un claro impacto en la calidad del sonido y, en consecuencia, en la calidad de los servicios proporcionados por el intérprete (Kelly, 2008; Rosenberg, 2004; Lázaro Gutiérrez y Cabrera Méndez, 2019).

Las condiciones laborales de los intérpretes remotos son definitivamente distintas, y no solo por el uso de las tecnologías (Lázaro Gutiérrez, 2019). De hecho, la IR implica un nuevo concepto de los encargos de interpretación que también tiene consecuencias en cuanto a costes y precios. Es indudable que la IR supone un gran ahorro para el proveedor (y consumidor) del servicio. 
Los intérpretes remotos no incurren en gastos de desplazamiento ni de espera y, aunque muchas empresas de interpretación telefónica han establecido una asignación mínima por encargo e incluyen pagos extra mensuales por disponibilidad nocturna y en fin de semana y festivos, lo intérpretes remotos, por lo general, cobran por minutos. Desde las asociaciones profesionales de intérpretes, de hecho, se ha criticado esta merma en las condiciones profesionales (sobre todo, en comparación con las establecidas para la interpretación de conferencias), aduciendo que los intérpretes mejor cualificados no están dispuestos a trabajar por esos emolumentos y en esas condiciones, por lo que el servicio queda en manos de intérpretes peor preparados o menos experimentados. Desde las empresas de IR, se alega, en cambio, que los intérpretes remotos tienen la capacidad de aceptar más encargos en horarios diversos y, además, pueden compaginar la interpretación con otras actividades, como la traducción.

Por último, varios autores han mencionado la falta de especialización temática de los intérpretes remotos (Gracia-García, 2002). Esto se debe, en gran medida, a la variedad de clientes que las empresas de IR tienen y los diferentes protocolos de actuación que establecen con ellos. En parte para cubrir este vacío, las empresas de IR suelen formar a sus empleados tanto cuando se incorporan a la compañía como de manera continua por motivos de calidad y para adaptarse a trabajar con clientes nuevos (ver, por ejemplo, Jiménez Higuera, 2018).

\section{3. Principios generales de la formación en IR}

La formación en IR debe perseguir el desarrollo de las competencias necesarias para desempeñar dicha actividad de manera profesional. Para Skinner, Napier y Braun (2018: 18), todo el que quiera dedicarse a la IR debe reunir los siguientes requisitos: 1) una sólida competencia en interpretación ${ }^{3}$; 2) una amplia experiencia profesional en interpretación; 3) una amplia cultura general; 4) un amplio léxico en las dos lenguas de trabajo; y 5) un alto conocimiento intercultural. No obstante, a estas competencias, básicas para toda actividad interpretativa, se deben sumar las destrezas propias de la IR asociadas a la coordinación de la interacción, como la gestión del inicio del encuentro y de su finalización, la gestión de los turnos de palabra y la realización de interrupciones por parte del intérprete, además del dominio de los elementos prosódicos y el empleo de la primera y la tercera persona (Fernández Pérez, 2015: 261-262).

En cuanto a la metodología docente, parece existir cierto consenso entre los formadores en IB (y, por extensión, en IR) de utilizar los ejercicios de simulación o role-plays, debido a que colocan al estudiante en una situación de interpretación lo más próxima posible a la realidad, en la que tendrán que enfrentarse a retos lingüísticos, culturales e interaccionales como los que surgen en el ejercicio profesional (Niemants y Cirillo, 2017: 12). Además de la simulación, los beneficios de estos ejercicios pedagógicos residen en la contextualización previa del encargo (conocida como

Entre los elementos que componen la macrocompetencia en interpretación destacan la competencia lingüística en el par de lenguas de trabajo, la competencia cultural e intercultural, competencia temática (del ámbito específico en que se desarrolle la interacción) y la competencia en transferencia lingüística o translativa, además de las habilidades relativas a la gestión de la comunicación y a la deontología (Pöchhacker, 2007: 166 y ss.). 
briefing del intérprete) y, sobre todo, en la posterior puesta en común (debriefing) por parte de todos los que hayan participado en la simulación, no solo del estudiante que haya actuado como intérprete, sino del resto de estudiantes que la hayan observado y evaluado. Debido a la infraestructura técnica necesaria, es difícil incluir la enseñanza de la IR en un entorno habitual de formación de intérpretes. Sin embargo, Fantinuoli y Prandi (2018: 172-173) sostienen que hay determinadas actividades sencillas que pueden resultar muy ventajosas para los estudiantes, como el estudio de la actividad en el mercado laboral, el análisis de estudios empíricos realizados o las simulaciones de interpretación por videoconferencia realizadas a través de programas como Skype.

Como expone Fernández Pérez (2015: 263-264), estos ejercicios de simulación pueden producirse de dos modos: a partir de guiones previamente redactados o mediante intervenciones improvisadas en función de una serie de directrices. A pesar de que los ejercicios guionizados tienen la desventaja de la falta de naturalidad en el discurso (se corre el riesgo de terminar leyendo y no oralizando la simulación), son muy ventajosos porque permiten al docente un mayor control de la actividad y una mayor secuenciación y gradación de los objetivos de aprendizaje en comparación con las simulaciones improvisadas. Este segundo tipo de simulación, además, requiere una mayor capacidad de improvisación por parte de quien represente los papeles asignados y produce un discurso más fragmentado, incoherente y repetitivo, que puede poner al intérprete novel en dificultades que, en un primer estadio, no resulten de especial interés formativo o incluso desmotivadoras.

\section{DESCRIPCIÓN DE LA EXPERIENCIA DOCENTE UPO-UAH}

\subsection{Justificación de la experiencia docente}

La adaptación al marco del EEES supuso un cambio profundo de los estudios universitarios de nuestro país, no solo en cuanto a su estructura formal, sino sobre todo en lo referente a la metodología. Como consecuencia, el diseño de los programas de las asignaturas de los nuevos grados, así como el papel del profesor en la enseñanza universitaria, han cambiado sustancialmente.

En la formación en interpretación, y en IB en concreto, en la clase tradicional, la docencia solía basarse ya en la interpretación realizada por el docente, que era tomada como el patrón de competencia que debían seguir los intérpretes en formación, ya en la evaluación que el docente realizaba de la interpretación que un alumno llevaba a cabo. En la nueva docencia centrada en el alumno, en cambio, se fomenta la adquisición de competencias no solo mediante la práctica de los intérpretes en formación sino también por medio de la observación de situaciones reales de interpretación, de la discusión sobre actuaciones específicas y de la coevaluación (Niemants y Cirillo, 2017: 12).

Como se ha mencionado anteriormente, la IR está cada vez más demandada en nuestros días gracias a las múltiples ventajas que presenta respecto a la interpretación presencial en muchos contextos. Por ello, no puede cuestionarse la pertinencia de que las universidades den respuesta a esta nueva realidad ofreciendo a los futuros intérpretes profesionales formación en esta modalidad de interpretación, que puede suponer una importante salida laboral (García Luque, 2009: 27), y propiciando el adecuado desarrollo de las competencias asociadas a la IR. Sin embargo, curiosamente, 
la IR no parece merecer atención en los actuales programas universitarios de formación de intérpretes, que no incluyen, no ya asignaturas concretas, sino objetivos formativos específicos en IR (Ruiz Mezcua, 2018: 14).

Este era el caso de los programas de grado de nuestras dos universidades, la UPO y la UAH, a pesar de ser centros destacados de formación en Traducción e Interpretación. En un intento por salvar este vacío formativo y proporcionar a los estudiantes de la UPO y la UAH experiencias formativas en esta modalidad interpretativa específica se diseñó un taller docente interuniversitario en IR, que se describe en mayor profundidad en los siguientes apartados.

\subsection{Contexto formativo}

Cabe esbozar el contexto formativo-curricular en que se ha desarrollado el taller, que se sintetiza en la Tabla 1. Por un lado, los estudiantes de la UPO que han participado en esta experiencia se encontraban cursando la asignatura Interpretación Bilateral B/A/B Inglés/Español/ Inglés, que, como se plasma en su guía docente ${ }^{4}$, persigue "introducir al estudiante en la práctica y en los aspectos teóricos y profesionales más relevantes de la interpretación bilateral". Se trata de una materia obligatoria, de 6 créditos ECTS, impartida en el segundo cuatrimestre de $4^{\circ}$ del Grado en Traducción e Interpretación (Inglés), titulación que "pretende dar respuesta a los nuevos retos sociales y culturales del mundo actual, formando a los futuros profesionales de la traducción e interpretación de manera competitiva"s. Se trata, por tanto, de unos estudios específicamente diseñados para dotar a sus egresados con las competencias requeridas para ejercer la profesión de traductor $\mathrm{y} / \mathrm{o}$ intérprete de manera eficaz.

Por otro lado, los alumnos de la UAH que participan en esta actividad pertenecen a la asignatura Introducción a la Interpretación. Se trata de una asignatura optativa de 8 créditos ECTS que se imparte en el segundo cuatrimestre del Grado en Lenguas Modernas y Traducción, que, implantado desde el curso 2009-2010, persigue el objetivo de formar a profesionales cualificados capaces de ayudar a superar fronteras lingüísticas en los ámbitos político, económico, social y cultural. Las enseñanzas del grado están diseñadas para proporcionar una formación adecuada tanto en los aspectos teóricos como en los aplicados a la traducción, aportando asimismo nociones sobre la interpretación, limitándose, de hecho, a esta asignatura optativa que se oferta para los alumnos de tercero y cuarto cursos y cuyos objetivos son, precisamente, proporcionar una introducción a la interpretación como forma de mediación interlingüística e intercultural oral.

Disponible en: https://cutt.ly/vexpTuT [Consulta: 1-9-2019].

5 Informe VERIFICA del Grado en Traducción e Interpretación (Inglés). Disponible en: https://cutt.ly/ gexpZ40 [Consulta: 5-9-2019]. 


\begin{tabular}{|l|l|l|}
\hline & \multicolumn{1}{|c|}{ Asignatura UPO } & \multicolumn{1}{c|}{ Asignatura UAH } \\
\hline Titulación & Grado en Traducción e Interpretación & Grado en Lenguas Modernas y Traducción \\
\hline Curso & $4^{\circ}$ & $3^{\circ} \mathrm{y} 4^{\circ}$ \\
\hline ECTS & 6 & 8 \\
\hline Naturaleza & Obligatoria & Optativa \\
\hline$N^{\text {o }}$ alumnos & 60 (en Línea 1) y 60 (en Línea 2) & 20 \\
\hline Objetivo principal & $\begin{array}{l}\text { Introducir a la práctica y a los aspectos } \\
\text { teóricos y profesionales más relevantes de la la } \\
\text { interpretación bilateral }\end{array}$ & $\begin{array}{l}\text { Introducción a la interpretación como } \\
\text { forma mediación interlingüística e } \\
\text { intercultural oral. }\end{array}$ \\
\hline $\begin{array}{l}\text { Relación con otras } \\
\text { asignaturas afines }\end{array}$ & $\begin{array}{l}\text { Impartida después de otra asignatura } \\
\text { obligatoria sobre técnicas de interpretación } \\
\text { y en el mismo cuatrimestre que otra optativa } \\
\text { de interpretación de conferencias }\end{array}$ & $\begin{array}{l}\text { Única asignatura sobre interpretación } \\
\text { presente en la titulación }\end{array}$ \\
\hline
\end{tabular}

Tabla 1. Comparativa entre UPO y UAH.

\subsection{Diseño, metodología e implementación}

En los tres cursos consecutivos de 2016-17, 2017-18 y 2018-19 se ha llevado a cabo una actividad formativa introductoria a modo de taller de IR con el fin de que los futuros egresados de la UPO y de la UAH, por un lado, conozcan esta novedosa modalidad de interpretación y, por otro lado, tengan una experiencia formativa en este ámbito que les permita reflexionar acerca de las competencias relativas a la IR. Esta experiencia docente surgió de la iniciativa de los autores de esta contribución, docentes de interpretación en sus universidades, por ofrecer una experiencia de enseñanza-aprendizaje real en IR.

Para el diseño de esta experiencia, se tuvieron en cuenta los siguientes condicionantes causados por el desigual contexto formativo, esbozado en el apartado anterior. Si bien se partía de la ventaja de que ambas materias (tanto la de la UPO como la de la UAH) se imparten en el segundo cuatrimestre y en la misma combinación lingüística (español-inglés), los contextos curriculares de las asignaturas mostraban diferencias notables. Para empezar, los estudiantes de la UPO cursan en el primer cuatrimestre de su $4^{\circ}$ curso de grado otra asignatura obligatoria de interpretación, denominada Técnicas de Interpretación B (Inglés), de 6 créditos ECTS, por lo que ya han recibido formación previa en habilidades básicas como la escucha atenta, el análisis del discurso oral y la toma de notas. Además, en la propia asignatura Interpretación Bilateral B/A/B Inglés/Español/Inglés se va formando a los estudiantes en competencias básicas de esta modalidad interpretativa, como el uso de la primera persona o la capacidad de petición de aclaraciones.

En cambio, la asignatura Introducción a la Interpretación supone para los estudiantes de la UAH su primer (y único) contacto formativo con la teoría y práctica interpretativas durante sus estudios de grado. Asimismo, la naturaleza de las asignaturas (obligatoria en el caso de la UPO y optativa en la UAH) también condiciona el desarrollo de la actividad docente. Así, mientras que para la asignatura de la UPO se ofertan, por línea, un total de 60 plazas (con años en los que el número 
de matriculados se acerca a la setentena, debido a dobles matrículas y otras circunstancias, como estudiantes de programas de movilidad), para la asignatura de la UAH se ofertan 20 plazas y suelen matricularse alrededor de 15 estudiantes.

Así pues, se decidió canalizar nuestra iniciativa docente en forma de taller de la siguiente manera: 1) se celebraría a partir de la décima semana de docencia, con el fin de que los estudiantes de la UAH hubieran recibido ya formación introductoria sobre interpretación y que no hubiera un desequilibrio competencial demasiado evidente entre los estudiantes de la UPO y los de la UAH; 2) se integraría en el horario habitual de los estudiantes de la UAH, lo que le confiere un carácter obligatorio para ellos, mientras que, debido al elevado número de estudiantes de la UPO, para estos últimos tendría carácter voluntario y extracurricular, con un número máximo de 20 plazas (por lo que tampoco podía tener una duración continuada); 3) el taller tendría un carácter introductorio, de aproximación a la modalidad de la IR, y no una ambición profesionalizante, con una duración final de 2 horas presenciales, que incluyeran una introducción teórica a la IR (30 minutos) y prácticas de IR (90 minutos); y 4) de entre las submodalidades de IR, se ha optado tanto por la interpretación telefónica como por la de videoconferencia, y nunca en situaciones de llamada a tres, sino siempre estando en la misma ubicación dos de los tres participantes (ya fuera uno de los usuarios y el intérprete o bien los dos usuarios), para evitar un mayor número de potenciales problemas, tanto de gestión de la comunicación como de carácter tecnológico.

En cuanto a la metodología docente, se decidió emplear diferentes herramientas y enfoques docentes. Se eligió la clase magistral por videoconferencia, seguida de un turno de debate para la introducción teórica tanto a la IR como a la IB. Para la parte práctica, se optó por la simulación de dos encargos de IR mediante role-plays guionizados, tanto en el ámbito sanitario como en el policial, por ser dos de los contextos en que con mayor frecuencia se recurre a la IR en el ámbito de la interpretación en los servicios públicos ${ }^{6}$ (Valero Garcés, 2008). Las simulaciones se irían alternando, de tal modo que un estudiante de la UPO tuviera que hacer de intérprete de manera remota para que, a continuación, en la siguiente situación, fuera un estudiante de la UAH quien interviniera como intérprete. También se alternarían las submodalidades: si en el primer role-play el intérprete mediaba a través del teléfono, en el segundo la interacción tenía lugar mediante videoconferencia. A continuación, se realizaría una puesta en común y un debate posterior a la simulación (debriefing) sobre la actuación del intérprete y cuantos elementos hayan llamado la atención de todos los participantes de la experiencia docente, con el ánimo de fomentar la observación y evaluación por pares. Por último, se planteó un debate final sobre la experiencia y la IR en general.

Este taller se ha realizado en tres cursos consecutivos (2016-17, 2017-18 y 2018-19) de manera muy similar. Los estudiantes de la UAH se encontraban en su aula habitual, el laboratorio de idiomas del Campus de Guadalajara, mientras que los estudiantes de la UPO se encontraban físicamente en el Aula de Telepresencia, una infraestructura docente especialmente acondicionada

6 Por ejemplo, en el curso 2016-17, el encargo de policial consistía en la presentación de una denuncia en una comisaría sevillana por parte de un ciudadano norteamericano al que le habían robado la cartera, mediada por un intérprete que se comunicaba a través de videoconferencia; en el curso 2018-19, el encargo de sanitaria consistía en la consulta entre una paciente que se comunicaba desde su centro de salud por videoconferencia con un médico especialista que se encontraba, junto con una intérprete, en otro centro sanitario. 
para las realización de videoconferencias disponible en su campus de Sevilla, y se establecía comunicación por medio del programa Skype. Tras presentarse y confirmar que la comunicación era posible, se procedía a las presentaciones teóricas, sobre la IR desde la UAH y sobre la IB desde la UPO (25 minutos en total), a las que seguía un breve turno de preguntas y respuestas (5 minutos).

A continuación, se pasaba a la parte práctica del taller. Un alumno de la UPO era seleccionado, de manera voluntaria, para hacer las veces de intérprete ante una situación determinada (por ejemplo, se le explicaba que acababa de recibir un encargo de interpretación para un centro de salud, en el que se encontraba un profesional sanitario - hispanoblante - y un paciente — angloparlante - y necesitaban su mediación para poder comunicarse), sin proporcionar una mayor contextualización, tal y como suele ocurrir en la vida real (González Rodríguez y Spinolo, 2017: 247). Seguidamente, tenía lugar la simulación guionizada mediada por el intérprete (ver ejemplo en Imagen 1). Una vez concluida la simulación, se realizaba un ejercicio de debriefing y puesta en común entre todos los participantes del role-play, con una mayor relevancia de lo manifestado por el intérprete y reforzada con las intervenciones de los demás alumnos que habían asumido una función de observadores y evaluadores tanto de la actuación del intérprete como de la situación en sí. Tras esta práctica, se pasaba a otra simulación guionizada, esta vez haciendo de intérprete un alumno de la UAH, siguiéndose el mismo procedimiento ya indicado, así cuantas diera tiempo a realizar de manera completa (por lo general, no menos de dos ni más de cuatro). Al final, se realizaba una última puesta en común a modo de síntesis acerca de la experiencia docente llevada a cabo ese día y sobre la IR en general (10-15 minutos) $)^{7}$.

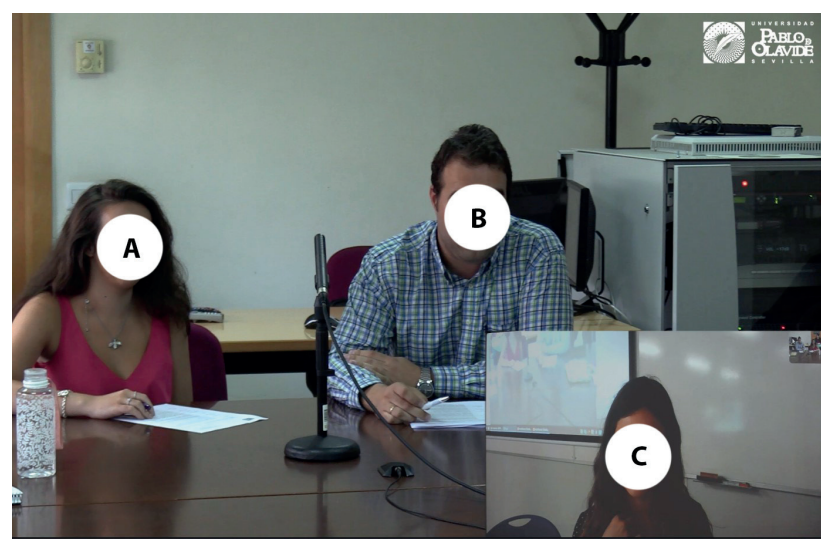

Imagen 1. Simulación de interpretación por videoconferencia ${ }^{8}$ (elaboración propia)

7 Las sesiones fueron grabadas en vídeo, con el fin de poder realizar análisis más detallados de determinados aspectos de la actuación de los intérpretes (por ejemplo, lenguaje no verbal, elementos prosódicos, uso de la primera/tercera persona, etc.), de los que, por motivos de espacio, no podemos dar cuenta en esta contribución.

8 En esta simulación, una ciudadana británica (A), interpretada por una estudiante británica de intercambio en la UPO, estaba cursando una denuncia por robo ante un agente de policía (B), interpretado por uno de los docentes, a través de una intérprete $(\mathrm{C})$ — una estudiante de la UAH — que prestaba su asistencia mediante videoconferencia. 


\subsection{Resultados}

El taller de IR que se describe en estas páginas es ya una actividad docente consolidada que se ha realizado en tres años consecutivos y se ha ido perfeccionando experiencia tras experiencia. Constituye un buen ejemplo de aprendizaje situado en tanto que parte del taller consiste en la simulación de encargos reales de IR, durante los que se replican situaciones (interacciones) que los alumnos tienen que abordar junto con sus dificultades asociadas, que hemos mencionado en apartados anteriores, como la falta o escasez de información visual (en telefónica y videoconferencia, respectivamente), la coordinación del discurso, el uso de la tecnología, los protocolos que se deben seguir con cada cliente, las condiciones laborales, el estrés emocional y la ética.

Todos los resultados que se enumeran en este apartado se han obtenido mediante el análisis de las grabaciones de las sesiones de debriefing y de síntesis realizadas tras las simulaciones, que, como se esperaba, han resultado una útil fuente de información retrospectiva por parte de los estudiantes. Gracias al análisis de lo manifestado por los participantes, se ha podido comprobar, por ejemplo, cómo los estudiantes experimentan en primera persona el impacto que la falta o la escasez de información visual (en el caso de la telefónica y de la videoconferencia respectivamente) tienen en la comprensión del discurso de los participantes.

También se ha podido constatar la influencia de la tecnología, que, a pesar de ser el elemento que hace posible el encuentro, plantea dificultades añadidas a las que hay que prestar atención, como la necesidad de comprobar que la conexión es adecuada y que todos los participantes son capaces de escucharse con nitidez (en más de una ocasión los estudiantes han mencionado cómo se tenía que repetir información durante la interacción al no haberse entendido a la primera). Sorprendentemente, algunos estudiantes, en cambio, afirmaron preferir la interpretación telefónica a la videoconferencia ya que no se exponían tanto a los interlocutores y no tenían que controlar tanto su expresión no verbal.

Dentro de la labor de coordinación de los intérpretes, los estudiantes han podido aprender técnicas para organizar la interacción y el discurso de los participantes principales del encuentro sin necesidad de apoyarse en el lenguaje no verbal (por ejemplo, en una de las simulaciones, uno de los interlocutores - interpretado por un docente - interrumpía al intérprete intencionadamente para ver cómo reaccionaba y qué técnicas de gestión de la comunicación utilizaba para que no se perdiese parte del contenido). De esta manera, los estudiantes han aprendido, por ejemplo, a interrumpir para pedir aclaraciones, para solicitar a los hablantes principales una pausa para poder interpretar o para recordarles las pautas básicas para el desarrollo de la conversación.

La variedad temática de los role-plays propuestos da pie, además, a explicar distintos protocolos que se utilizan dentro de la IR, como los que se aplican en casos de violencia de género. Asimismo, se han explorado aspectos relativos a las condiciones laborales de los intérpretes, como pueden ser el estar sometidos, en ocasiones, a un elevado nivel de estrés y alto riesgo de impacto emocional, como mencionan siempre los alumnos después de un role-play sobre una situación de tensión emocional para los interlocutores (por ejemplo, cuando la paciente se derrumba al reconocer que sus lesiones son consecuencia de episodios violentos por parte de su pareja). Cabe destacar, además, que los estudiantes destacan positivamente el abordaje práctico que este taller permite hacer 
en cuanto a aspectos éticos aplicados a la IR, como la presentación del intérprete ante los hablantes principales, durante la que el intérprete expone que las interpretaciones serán fieles y confidenciales, o la reiteración de la posición de neutralidad del intérprete ante determinadas posiciones (por ejemplo, en uno de los role-plays trabajados, el policía realiza una serie de desafortunados comentarios sobre la nacionalidad del denunciante, ante lo cual algunos intérpretes le repiten que están obligados a trasladar toda la información a las dos partes mientras que otros no dicen nada al respecto, poniendo así en entredicho su neutralidad).

Como mencionábamos anteriormente, a lo largo de los tres años en que se ha llevado a cabo esta experiencia docente, hemos sido capaces de introducir mejoras en su diseño e implementación. Por ejemplo: se han ido introduciendo elementos clave en los role-plays que sirvieran de pie para abordar aspectos relacionados con el trasvase de la información y con la ética profesional; se han plasmado en las introducciones teóricas sobre la IB y la IR aspectos que habían surgido en los momentos de debate y puesta en común con los alumnos de ediciones anteriores; $\mathrm{y}$, en el desarrollo de los role-plays, se ha decidido limitar las actuaciones de los alumnos a su función como intérpretes y dejar en manos de los docentes la representación de los papeles de los oradores principales ${ }^{9}$.

Por último, a partir de lo expresado por los propios estudiantes, se ha podido confirmar el gran interés que la actividad ha generado entre ellos, fomentando de forma destacada su motivación en el estudio de la interpretación en general y la IR en concreto, y en el posible desempeño de la IR como actividad profesional futura. Si bien han existido desniveles entre las actuaciones de los alumnos de la UPO y la UAH debidos a la menor preparación y exposición a la interpretación de los alumnos de la UAH, la actividad ha aportado a ambos grupos conocimientos teórico-prácticos que les han permitido realizar un aprendizaje significativo.

\section{CONCLUSIONES}

La Universidad debe dar respuesta a las demandas de la sociedad. La sociedad actual, dominada por el uso de las tecnologías de la información y la comunicación, está dando paso a nuevas modalidades de trabajo telemático, como la IR en el ámbito de la mediación entre lenguas y culturas. Por lo tanto, la realización de actividades docentes como la que se presenta en estas páginas es fundamental para complementar la formación en interpretación que se ofrece a nivel de grado en la universidad, centrada en modalidades interpretativas tradicionales.

El planteamiento metodológico, diseñado en torno a tres pilares fundamentales (clase magistral introductoria, role-plays y reflexión conjunta), ha resultado eficaz para la adquisición

En la primera edición de nuestra experiencia (curso 2016-17), la simulación contó con la actuación de estudiantes no solo como intérpretes sino también representando los papeles de los oradores principales del guion, como algunos autores defienden (Fernández Pérez, 2015: 263). Sin embargo, observamos que los estudiantes que representaban dicho papel no seguían tanto la actuación del intérprete sino que, más bien, se ceñían exclusivamente a las intervenciones redactadas en el roleplay. Por ello, decidimos que sería mejor que fueran los dos docentes, con experiencia en la enseñanza de la interpretación y mayor capacidad para improvisar, o al menos uno de ellos en todo caso, quienes hicieran de oradores originales, como recomiendan otros autores siempre que sea posible (Ozolins, 2017: 55). 
de competencias relacionadas con la IR, tal y como se ha podido comprobar a partir del análisis de las grabaciones de las sesiones. Evidentemente, la duración limitada de este taller no da a lugar a perseguir un objetivo plenamente profesionalizante, pero esta experiencia docente interuniversitaria sí permite la inclusión de contenidos y desarrollo de competencias adaptados a esta nueva actividad profesional de forma introductoria.

\section{REFERENCIAS BIBLIOGRÁFICAS}

Bot, H. (2005). Dialogue interpreting as a specific case of reported speech. Interpreting, 7(2), 237261. DOI: https://doi.org/10.1075/intp.7.2.06bot

Braun, S. (2006). Multimedia communication technologies and their impact on interpreting. En H. Gerzymisch-Arbogast (Ed.), Proceedings of the Marie Curie Euroconferences MuTra: Audiovisual translation scenarios Copenhagen, s. p. Disponible en: http://epubs.surrey. ac.uk/804844/ [Consulta: 1-9-2019].

Cheng, Q. (2015). Examining the challenges for telephone interpreters in New Zealand. Tesis: Auckland University of Technology. Disponible en: https://core.ac.uk/download/pdf/56365377. pdf [Consulta: 1-8-2019].

Collados Aís, Á., Fernández Sánchez, M. M. y De Manuel Jerez, J. (2001). La Interpretación Bilateral: Características, situaciones comunicativas y modalidades. En Á. Collados Aís y M. M. Fernández Sánchez (Coords.), Manual de interpretación bilateral (pp. 61-77). Granada: Comares.

Collados Aís, Á. y Fernández Sánchez, M. M. (2001) (Coords.). Manual de interpretación bilateral. Granada: Comares.

Crezee, I. (2013). Introduction to healthcare for interpreters and translators. Amsterdam: John Benjamins. DOI: https://doi.org/10.1075/z.181

Fantinuoli, C. y Prandi, B. (2018). Teaching information and communication technologies. A proposal for the interpreting classroom. Trans-kom, Journal of Translation and Technical Communication Research, 11(2), 162-182. Disponible en http://www.trans-kom.eu/bd11nr02/transkom_11_02_02_Fantinouli_Prandi_Teaching.20181220.pdf [Consulta: 1-9-2019].

Fernández Pérez, M. M. (2015). Propuestas de ejercicios de simulación para la didáctica de la interpretación telefónica. MonTI, Monografías de Traducción e Interpretación, (2), 259-279. DOI: https://doi.org/10.6035/MonTI.2015.ne2.10

Fernández Pérez, M. M. (2017). Interaction Management Skills in Telephone Interpreting. Revista Canaria de Estudios Ingleses, (75), 103-117. Disponible en: https://riull.ull.es/xmlui/handle/915/6968 [Consulta: 1-8-2019].

Foulquié Rubio, A. I. (2019). Interpretación en los servicios públicos y en la empresa. Murcia: Servicio de Publicaciones de la Universidad de Murcia.

García Luque, F. (2009). La interpretación telefónica en el ámbito sanitario: realidad social y reto pedagógico. REDIT - Revista Electrónica de Didáctica de la Traducción y la Interpretación, (3), 18-30. DOI: https://doi.org/10.24310/REDIT.2009.v0i3.1920 
González Rodríguez, M. J. y Spinolo, N. (2017). Telephonic dialogue interpreting. A short teaching course. En L. Cirillo y N. Niemants (Eds.), Teaching Dialogue Interpreting (pp. 241-257). Ámsterdam: John Benjamins. DOI: https://doi.org/10.1075/btl.138.12gon

Gracia-García, R. A. (2002). Telephone interpreting: A review of pros and cons. En S. Brennan (Ed.), Proceedings of the 43rd Annual Conference (pp. 195-216). Alexandria: American Translators Association.

Hsieh, E. (2006). Understanding medical interpreters: Reconceptualizing bilingual health communication. Health Communication, 20(2), 177-186. DOI: https://doi.org/10.1207/ s15327027hc2002 9

Jiménez Higuera, S. (2018). Specific Training for Telephone Interpreters, by Interpret Solutions. En A. Ruiz Mezcua (Ed.), Approaches to Telephone Interpretation. Research, Innovation, Teaching and Transference (pp. 51-59). Berna: Peter Lang.

Kelly, N. (2008). Telephone interpreting: A comprehensive guide to the profession. Victoria: Trafford.

Ko, L. (2006). The need for long-term empirical studies in remote interpreting research: A case study of telephone interpreting. Linguistica Antverpiensia, (5), 325-338. Disponible en https://lanstts.uantwerpen.be/index.php/LANS-TTS/article/view/167 [Consulta: 1-8-2019].

Lázaro Gutiérrez, R. (2019). Telephone interpreting and roadside assistance. Translation and Translanguaging in Multilingual Contexts, 5(3), 215-240. DOI: https://doi.org/10.1075/ ttmc.00033.laz

Lázaro Gutiérrez, R. y Cabrera Méndez, G. (2019). Context and pragmatic meaning in telephone interpreting. En P. Garcés-Conejos Blitvich, L. Fernández-Amaya y M. O. Hernández-López (Eds.), Technology Mediated Service Encounters (pp. 45-68). Ámsterdam: John Benjamins.

Lee, J. (2007). Telephone interpreting seen from the interpreters' perspective. Interpreting, 9(2), 231-252. DOI: https://doi.org/10.1075/intp.9.2.05lee

Mikkelson, H. (2003). Telephone interpreting: Boon or Bane? En L. Pérez González (Ed.), Speaking in tongues: Language across contexts and users (pp. 251-269). Valencia: Universitat de València.

Niemants, N. y Cirillo, L. (2017). Dialogue interpreting: Research, education and professional practice. En L. Cirillo y N. Niemants (Eds.), Teaching Dialogue Interpreting (pp. 2-25). Ámsterdam: John Benjamins. DOI: https://doi.org/10.1075/btl.138

Ozolins, U. (2017). It's not about the interpreter. Objectives in dialogue interpreting teaching. En L. Cirillo y N. Niemants (Eds.), Teaching Dialogue Interpreting (pp. 45-62). Ámsterdam: John Benjamins. DOI: https://doi.org/10.1075/btl.138.02ozo

Pertusa Elorriaga, I. (2012). La interpretación en la distancia. Suplemento: la traducción y la interpretación en los servicios públicos. Revista Traditori, (3), 25-29.

Pöchhacker, F. (2007) (2 ${ }^{\mathrm{a}}$ Ed.). Introducing Interpreting Studies. Londres: Routledge.

Rosenberg, B. A. (2004). A data driven analysis of telephone interpreting. En C. Wadensjö, B. E. Dimitrova y A-L. Nilsson (Eds.), The Critical Link 4: Professionalisation of interpreting in the community (pp. 65-77). Ámsterdam: John Benjamins. DOI: https://doi.org/10.1075/ btl.70.09ros 
Ruiz Mezcua, A. (2018). General Overview of Telephone Interpretation (TI): A State of the Art. En A. Ruiz Mezcua (Ed.), Approaches to Telephone Interpretation. Research, Innovation, Teaching and Transference (pp. 7-17). Berna: Peter Lang. DOI: https://doi.org/10.3726/b13326

Skinner, R., Napier, J. y Braun, S. (2018). Interpreting via video link: Mapping the field. En J. Napier, R. Skinner y S. Braun (Eds.), Here or There: Research on Interpreting via Video Link (pp. 11-35). Washington DC: Gallaudet.

Valero Garcés, C. (2008). Formas de mediación intercultural: Traducción e Interpretación en los Servicios Públicos. Conceptos, datos, situaciones y práctica. Granada: Comares.

Wadensjö, C. (1999). Telephone interpreting and the synchronization of talk in social interaction. The Translator, 5(2), 247-264. DOI: https://doi.org/10.1080/13556509.1999.10799043 